\title{
Squamous Cell Carcinoma of Renal Pelvis: Can Calculi Be Life Threatening?
}

\author{
Harsh Batra, Charanjeet Ahluwalia*, Amit Yadav and Indrani Dhawan
}

Department of Pathology and Lab Medicine, VMMC and Safdarjung Hospital, New Delhi, India

\section{Dear Sir}

Primary squamous cell carcinoma (SCC) of renal pelvis has a variable incidence of about $0.5-15 \%$ of all urothelial

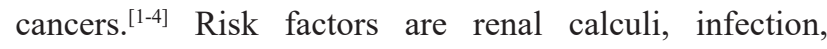
endogenous and exogenous chemicals, hormonal imbalance, radiotherapy and vitamin A deficiency. ${ }^{[5]} \mathrm{We}$ report a case of primary SCC of left renal pelvis.

A 40-year old male presented with dull left sided flank pain, fever and hematuria. Urine examination showed red colored urine. There was $2+$ proteinuria. Microscopy revealed 15-20 RBCs/hpf and 5-7 pus cells/hpf. Kidney function tests revealed a serum creatinine level of $0.97 \mathrm{mg} /$ $\mathrm{dl}$ and blood urea levels of $21.82 \mathrm{mg} / \mathrm{dl}$. There was a past history of left pyelolithotomy about 7 years back.

CECT KUB showed enlarged left hydronephrotic kidney measuring $21 \mathrm{~cm}$ (longitudinal) showing heterogeneously enhancing lesion with non-enhancing hypodense areas measuring $8 \times 7.3 \times 8.2 \mathrm{~cm}$ (AP X TR X CC) (Fig. 1) Lesion was seen extending up to left proximal ureter. Multiple calculi in pelvicalyceal system were noted, largest measuring $5.5 \mathrm{~mm}$.

Left sided nephrectomy was performed, and specimen of already cut open left radical nephrectomy measuring $16 \times 3 \times 8 \mathrm{~cm}$ with dilated pelvicalyceal system was received in the histopathology lab. Pelvis showed a grey white friable tumor with areas of necrosis measuring $10 \times 9 \times 6 \mathrm{~cm}$. Focal capsular breech was noted with perinephric fat involvement.

Histopathology examination showed moderately differentiated squamous cell carcinoma of left renal pelvis involving parenchyma, breeching capsule and extending into the perinephric fat (Fig. 2\&3). Sections from the ureter also showed deposits of the same tumor. All lymph nodes dissected were free of tumor.

A final diagnosis of moderately differentiated squamous cell carcinoma of left renal pelvis, stage pT4N0MX was given. The patient refused adjuvant chemotherapy and radiotherapy and was discharged on $6^{\text {th }}$ post-operative day. On follow up patient was found to have metastasis in liver.
Primary SCC of renal pelvis constitute only about $0.7-7 \%$ of all primary malignant tumors of the renal pelvis. ${ }^{[6]} \mathrm{A}$ female sex predilection is seen with most of the patients being in the age group between $50-70$ years. ${ }^{[7]}$

A diagnosis of primary squamous cell carcinoma of renal pelvis was made in our case, only after extensive sampling to exclude any urothelial component, and the tumor showed extensive infiltration into the renal parenchyma. The closest differential diagnosis is urothelial carcinoma with squamous differentiation.

The major etiological factors are history of associated renal calculi and infections.[5] The metaplastic pathophysiology of the SCC developing in renal pelvis remains debatable, as some studies have also shown the occurrence in absence of etiological factors.[4] In our case, the patient had renal calculi with a past history of pyelolithotomy, thus reiterating the importance of this etiological agent, which might have led to metaplasia, which subsequently must have proceeded to squamous cell carcinoma.

Surgery is the mainstay of treatment with adjuvant chemotherapy offering only a marginal relief. Primary SCC of renal pelvis are associated with a very poor prognosis, with presentation at advanced stages, and very few patients surviving for 5 years. $[4,8]$

Our patient, after initial surgery, refused for any adjuvant therapy and has presently developed extensive metastasis in liver.

To concluded, long standing, large renal calculi are associated in the etiology of these tumors. These tumors are aggressive, often presenting at late stages and offer a dismal prognosis. Thus, history of long standing calculi is important while diagnosing these tumors. Also, even non-obstructive calculi should be treated promptly so as to reduce the risk of progressing to tumor. Surgery being the mainstay mode of treatment in these tumors, can offer partial relief to the patient, provided, the disease is localized, but as most of the cases present at an advanced stage, treatment options offer less benefit to the patient. 


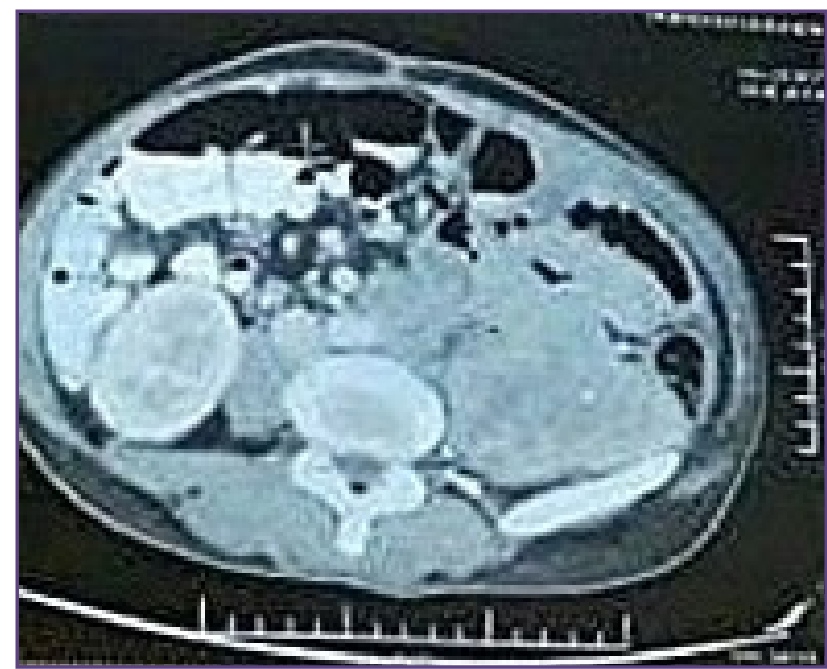

Fig. 1: CECT showing enlarged left kidney with heterogenous lesion.

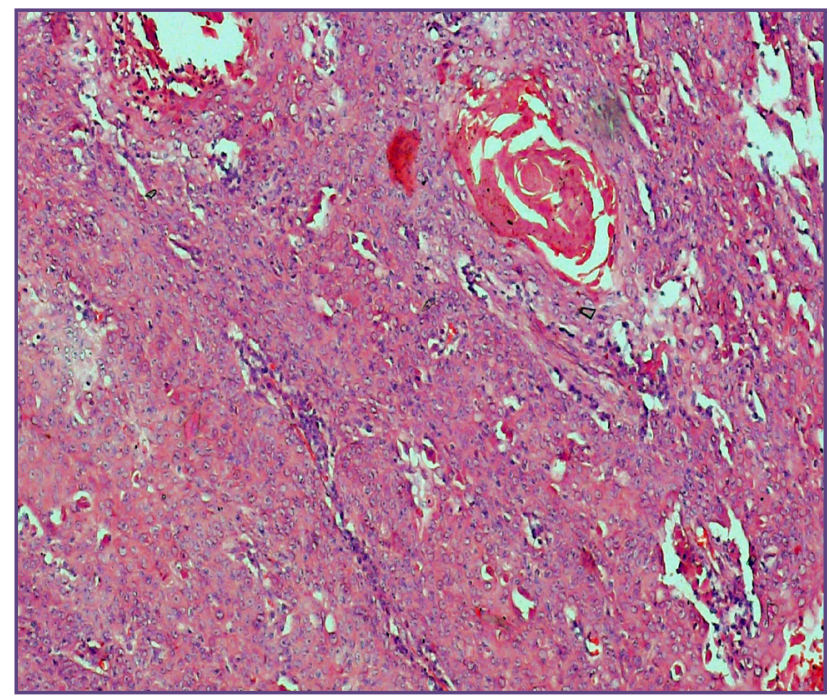

Fig. 3: Tumor arranged in sheets showing moderately differentiated tumor cells with a focus of squamous pearl formation(arrow). (H\&E, 100X).

\section{Reference}

1. Blacher EJ, Johnson DE, Abdul-Karim FW, Ayala AG. Squamous cell carcinoma of renal pelvis. Urology. $1985 \mathrm{Feb}$ $1 ; 25(2): 124-6$.

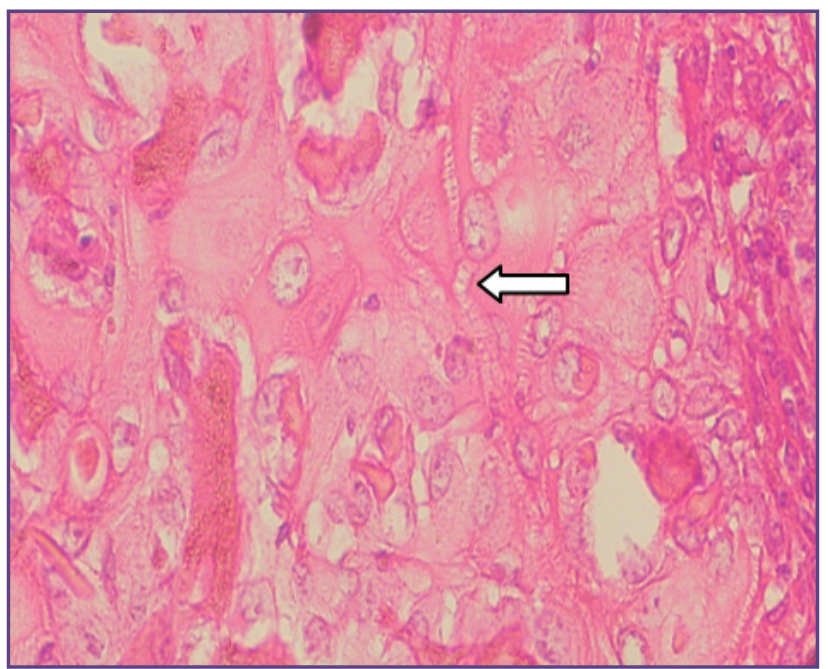

Fig. 2: Tumour cells showing intercellular bridges (arrow). (H \& E, 400X).

2. Li MK, Cheung WL. Squamous cell carcinoma of the renal pelvis. The Journal of urology. 1987 Aug 1;138(2):269-71.

3. Lee TY, Ko SF, Wan YL, Cheng YF, Yang BY, Huang DL, Hsieh HH, Yu TJ, Chen WJ. Renal squamous cell carcinoma: CT findings and clinical significance. Abdom Imaging. 1998 Mar 1;23(2):203-8.

4. Holmäng S, Lele SM, Johansson SL. Squamous cell carcinoma of the renal pelvis and ureter: Incidence, symptoms, treatment and outcome. J Urol. 2007;178:51-6.

5. Bandyopadhyay R., Biswas S., Nag D. Squamous cell carcinoma of the renal pelvis presenting as hydronephrosis. J Cancer Res Ther. 2010;6:537-539.

6. Mizusawa H, Komiyama I, Ueno Y, Maejima T, Kato H. Squamous cell carcinoma in the renal pelvis of a horseshoe kidney. Int J Urol 2004;11:782-4

7. Talwar N, Dargan P, Arora MP, Sharma S, Sen AK. Primary squamous cell carcinoma of the renal pelvis masquerading as pyonephrosis: A case report. Indian J Pathol Microbiol 2006;49:418-20.

8. Busby JE, Brown GA, Tamboli P, et al. Upper urinary tract tumors with nontransitional histology: a single-center experience. Urology. 2006;67(3):518-523.

\section{*Corresponding author:}

Dr. Charanjeet Ahluwalia, A-15/1, Vasant Vihar, New Delhi-110057 India

Email: charanjeet.ahluwalia@rediffmail.com

Financial or other Competing Interests: None. 